\title{
IIA/IIB supergravity and ten-forms
}

\author{
E.A. Bergshoeff, ${ }^{a}$ J. Hartong, ${ }^{b}$ P.S. Howe, ${ }^{c}$ T. Ortín ${ }^{d}$ and F. Riccioni ${ }^{c}$ \\ ${ }^{a}$ Centre for Theoretical Physics, University of Groningen, \\ Nijenborgh 4, 9747 AG Groningen, The Netherlands \\ ${ }^{b}$ Albert Einstein Center for Fundamental Physics, Institute for Theoretical Physics, \\ University of Bern, \\ Sidlerstrasse 5, 3012 Bern, Switzerland \\ ${ }^{c}$ Department of Mathematics, King's College London, \\ Strand London WC2R 2LS U.K. \\ ${ }^{d}$ Instituto de Física Teórica, UAM/CSIC, Facultad de Ciencias C-XVI, \\ C.U. Cantoblanco, E-28049-Madrid, Spain \\ E-mail: E.A.Bergshoeff@rug.nl, hartong@itp.unibe.ch, \\ Paul.Howe@kcl.ac.uk, Tomas.Ortin@uam.es, Fabio.Riccioni@kcl.ac.uk
}

ABSTRACT: We perform a careful investigation of which $p$-form fields can be introduced consistently with the supersymmetry algebra of IIA and/or IIB ten-dimensional supergravity. In particular the ten-forms, also known as "top-forms", require a careful analysis since in this case, as we will show, closure of the supersymmetry algebra at the linear level does not imply closure at the non-linear level. Consequently, some of the (IIA and IIB) ten-form potentials introduced in earlier work of some of us are discarded. At the same time we show that new ten-form potentials, consistent with the full non-linear supersymmetry algebra can be introduced. We give a superspace explanation of our work. All of our results are precisely in line with the predictions of the $E_{11}$ algebra.

KEYwords: p-branes, Extended Supersymmetry, Superspaces, Gauge Symmetry

ARXIV EPRINT: 1004.1348 


\section{Contents}

1 Introduction 1

2 The top-forms of IIB supergravity 2

2.1 IIB supergravity 2

2.2 Ten-form potentials 5

$\begin{array}{lll}2.3 & \text { IIB superspace } & 7\end{array}$

3 The top-forms of IIA supergravity $\quad 9$

3.1 IIA supergravity 9

$\begin{array}{ll}3.2 \text { Ten-form potentials } & 12\end{array}$

$\begin{array}{lll}3.3 & \text { IIA superspace } & 13\end{array}$

4 Conclusions $\quad 14$

\section{Introduction}

Supergravity theories provide important information about string theory. In particular, the $p$-form fields of the supergravity multiplet are in one-to-one correspondence, via their occurrence in the world-volume actions, with the branes of string theory provided that supersymmetry can be maintained. ${ }^{1}$ The $p$-form fields with $p \leq D-2$ can be easily predicted since they describe the physical states (or their duals) of the (D-dimensional) supergravity theory in question. This is not the case for the potentials of rank $p=D-1$ ("de-form" potentials) and rank $p=D$ ("top-form" potentials). A well-known example of a de-form potential is the 9-form potential [1] of massive IIA supergravity [2] that gives a dual description of the mass parameter $m$ present in the theory.

Ten-dimensional supergravities have been constructed a long time ago both for the non-chiral IIA case [3-5] as well as for the chiral IIB case [6-8]. A partially "democratic" formulation of these theories, where all the RR fields are introduced together with their magnetic duals, was given in [9] and [10]. This was then extended to a fully democratic formulation, including also the magnetic duals of the NS fields for both the IIA [11] and IIB [12] cases. This analysis also included a fairly complete discussion of the de-form and top-form potentials for IIA and IIB. A superspace interpretation of the latter case was given in [13].

It is the purpose of this note to reconsider the results of [11-13]. The reason for this is the following. In [12] we showed that an $\mathrm{SU}(1,1)$-doublet of ten-form potentials could

\footnotetext{
${ }^{1}$ An exception are those $p$-form fields that under supersymmetry do not transform into the gravitino. An example of this is the 8-form dual of the IIA dilaton, see the last line of eq. (3.4).
} 
be introduced, consistently with the lowest-order IIB supersymmetry algebra, with only trivial Abelian gauge transformations:

$$
\delta A_{10}^{\alpha}=d \Lambda_{9}^{\alpha}, \quad \alpha=1,2 .
$$

In contrast, both $E_{11}$ [14] and IIB superspace [13] considerations predict a doublet tenform potential that forms a non-trivial gauge algebra with the other $p$-forms of the theory. Moreover, as we will show in this paper, IIB superspace does not allow for two doublets. We will show that the different inconsistencies are resolved as follows. By performing an explicit check of the full non-linear supersymmetry algebra, we show that, whereas at the lowestorder level two doublets of ten-form potentials are allowed, this is no longer the case at the non-linear level: surprisingly, we find that the trivial doublet (1.1) is not consistent with the full IIB supersymmetry algebra. The same result applies to the IIA case. This is a rare example of a case that a result, established at the lowest-order level of the supersymmetry algebra, cannot be extended to the full non-linear level. It relies on the fact that topforms are special in the sense that a general coordinate transformation can be rewritten as a gauge transformation. Consequently, when closing the algebra at the lowest order in fermions one "only" has to make sure that the algebra closes up to gauge transformations while for the lower rank potentials one needs both gauge transformations and g.c.t.'s.

On the other hand, IIB superspace and $E_{11}$ considerations do predict the existence of a doublet of ten-form potentials with a non-trivial gauge-symmetry structure which were not found in [12]. We show that this doublet was missed because of a specific identity that was not used in the calculations. Here, we point out this identity and show that the supersymmetry algebra can now indeed be closed. For the IIA case we establish a similar result: we show that the trivial ten-form found in [11] does not persist at the non-linear level. Instead, by using a similar non-trivial identity as in the IIB case, we show that a new ten-form potential is allowed, with a non-trivial gauge algebra structure, that is consistent with the full non-linear IIA supersymmetry algebra.

This paper is organised as follows. In section 2 we first discuss the ten-form potentials of IIB supergravity. In section 3 we give a similar discussion of the IIA case. Finally, in section 4 we give our conclusions.

\section{The top-forms of IIB supergravity}

In this section we want to reconsider the analysis of ten-form potentials present in the IIB supergravity multiplet that was performed in [12]. We will first review the supersymmetry algebra and the algebra of gauge transformations for all the propagating fields. We will then consider in more detail the 10-forms. Finally, we will review the superspace results of [13].

\subsection{IIB supergravity}

The propagating fields of IIB supergravity and their magnetic duals are the vielbein $e_{\mu}{ }^{a}$, two scalars parametrising the symmetric manifold $\mathrm{SU}(1,1) / \mathrm{U}(1)$ and described in terms of the matrix $V_{ \pm}^{\alpha}$, where \pm denotes the $\mathrm{U}(1)$ charge and $\alpha$ is a doublet index of $\mathrm{SU}(1,1)$, a doublet of 2 -forms $A_{\mu_{1} \mu_{2}}^{\alpha}$ and a self-dual 4 -form $A_{\mu_{1} \ldots \mu_{4}}$ that is a singlet of $\mathrm{SU}(1,1)$, 
together with a doublet of 6 -forms $A_{\mu_{1} \ldots \mu_{6}}^{\alpha}$ and a triplet of 8 -forms $A_{\mu_{1} \ldots \mu_{8}}^{\alpha \beta}$. The gauge transformations of the form fields can be written in an abelian basis in which all gauge transformations commute, and in particular one gets

$$
\begin{aligned}
\delta A_{\mu_{1} \mu_{2}}^{\alpha} & =2 \partial_{\left[\mu_{1}\right.} \Lambda_{\left.\mu_{2}\right]}^{\alpha}, \\
\delta A_{\mu_{1} \ldots \mu_{4}} & =4 \partial_{\left[\mu_{1}\right.} \Lambda_{\left.\mu_{2} \ldots \mu_{4}\right]}-\frac{i}{4} \epsilon_{\gamma \delta} \Lambda_{\left[\mu_{1}\right.}^{\gamma} F_{\left.\mu_{2} \ldots \mu_{4}\right]}^{\delta} \\
\delta A_{\mu_{1} \ldots \mu_{6}}^{\alpha} & =6 \partial_{\left[\mu_{1}\right.} \Lambda_{\left.\mu_{2} \ldots \mu_{6}\right]}^{\alpha}-8 \Lambda_{\left[\mu_{1}\right.}^{\alpha} F_{\left.\mu_{2} \ldots \mu_{6}\right]}-\frac{160}{3} F_{\left[\mu_{1} \ldots \mu_{3}\right.}^{\alpha} \Lambda_{\left.\mu_{4} \ldots \mu_{6}\right]} \\
\delta A_{\mu_{1} \ldots \mu_{8}}^{\alpha \beta} & =8 \partial_{\left[\mu_{1}\right.} \Lambda_{\left.\mu_{2} \ldots \mu_{8}\right]}^{(\alpha \beta)}+\frac{1}{2} F_{\left[\mu_{1} \ldots \mu_{7}\right.}^{(\alpha} \Lambda_{\left.\mu_{8}\right]}^{\beta)}-\frac{21}{2} F_{\left[\mu_{1} \ldots \mu_{3}\right.}^{(\alpha} \Lambda_{\left.\mu_{4} \ldots \mu_{8}\right]}^{\beta)},
\end{aligned}
$$

where the corresponding gauge invariant field strengths are

$$
\begin{aligned}
& F_{\mu_{1} \ldots \mu_{3}}^{\alpha}=3 \partial_{\left[\mu_{1}\right.} A_{\left.\mu_{2} \mu_{3}\right]}^{\alpha}, \\
& F_{\mu_{1} \ldots \mu_{5}}=5 \partial_{\left[\mu_{1}\right.} A_{\left.\mu_{2} \ldots \mu_{5}\right]}+\frac{5 i}{8} \epsilon_{\alpha \beta} A_{\left[\mu_{1} \mu_{2}\right.}^{\alpha} F_{\left.\mu_{3} \ldots \mu_{5}\right]}^{\beta}, \\
& F_{\mu_{1} \ldots \mu_{7}}^{\alpha}=7 \partial_{\left[\mu_{1}\right.} A_{\left.\mu_{2} \ldots \mu_{7}\right]}^{\alpha}+28 A_{\left[\mu_{1} \mu_{2}\right.}^{\alpha} F_{\left.\mu_{3} \ldots \mu_{7}\right]}-\frac{280}{3} F_{\left[\mu_{1} \ldots \mu_{3}\right.}^{\alpha} A_{\left.\mu_{4} \ldots \mu_{7}\right]}, \\
& F_{\mu_{1} \ldots \mu_{9}}^{\alpha \beta}=9 \partial_{\left[\mu_{1}\right.} A_{\left.\mu_{2} \ldots \mu_{9}\right]}^{\alpha \beta}+\frac{9}{4} F_{\left[\mu_{1} \ldots \mu_{7}\right.}^{(\alpha} A_{\left.\mu_{8} \mu_{9}\right]}^{\beta)}-\frac{63}{4} F_{\left[\mu_{1} \ldots \mu_{3}\right.}^{(\alpha} A_{\left.\mu_{4} \ldots \mu_{9}\right]}^{\beta)} .
\end{aligned}
$$

The supersymmetry transformations of these gauge fields

$$
\begin{aligned}
\delta A_{\mu_{1} \mu_{2}}^{\alpha} & =\delta_{F} A_{\mu_{1} \mu_{2}}^{\alpha}, \\
\delta A_{\mu_{1} \ldots \mu_{4}} & =\delta_{F} A_{\mu_{1} \ldots \mu_{4}}-\frac{3 i}{8} \epsilon_{\gamma \delta} A_{\left[\mu_{1} \mu_{2}\right.}^{\gamma} \delta_{F} A_{\left.\mu_{2} \mu_{4}\right]}^{\delta}, \\
\delta A_{\mu_{1} \ldots \mu_{6}}^{\alpha} & =\delta_{F} A_{\mu_{1} \ldots \mu_{6}}^{\alpha}+40 A_{\left[\mu_{1} \ldots \mu_{4}\right.} \delta_{F} A_{\left.\mu_{5} \mu_{6}\right]}^{\alpha}-20 \delta_{F} A_{\left[\mu_{1} \ldots \mu_{4}\right.} A_{\left.\mu_{5} \mu_{6}\right]}^{\alpha}, \\
\delta A_{\mu_{1} \ldots \mu_{8}}^{\alpha \beta} & =\delta_{F} A_{\mu_{1} \ldots \mu_{8}}^{\alpha \beta}+\frac{21}{4} A_{\left[\mu_{1} \ldots \mu_{6}\right.}^{(\alpha} \delta_{F} A_{\left.\mu_{7} \mu_{8}\right]}^{\beta)}-\frac{7}{4} A_{\left[\mu_{1} \mu_{2}\right.}^{(\alpha} \delta_{F} A_{\left.\mu_{3} \ldots \mu_{8}\right]}^{\beta)}
\end{aligned}
$$

were derived in [12]. They have a particularly simple form, as pointed out in [11], in which all terms are at most linear in the gauge fields. Here we denote with $\delta_{F}$ the part of the supersymmetry transformation that only involves fermi bilinears, that are [12]

$$
\begin{aligned}
\delta_{F} A_{\mu_{1} \mu_{2}}^{\alpha} & =4 i V_{-}^{\alpha} \bar{\epsilon}^{*} \gamma_{\left[\mu_{1}\right.} \psi_{\left.\mu_{2}\right]}+V_{-}^{\alpha} \bar{\epsilon} \gamma_{\mu_{1} \mu_{2}} \lambda+\text { c.c. } \\
\delta_{F} A_{\mu_{1} \ldots \mu_{4}} & =\bar{\epsilon} \gamma_{\left[\mu_{1} \ldots \mu_{3}\right.} \psi_{\left.\mu_{4}\right]}+\text { c.c. } \\
\delta_{F} A_{\mu_{1} \ldots \mu_{6}}^{\alpha} & =12 V_{-}^{\alpha} \bar{\epsilon}^{*} \gamma_{\left[\mu_{1} \ldots \mu_{5}\right.} \psi_{\left.\mu_{6}\right]}+i V_{-}^{\alpha} \bar{\epsilon} \gamma_{\mu_{1} \ldots \mu_{6}} \lambda+\text { c.c. }, \\
\delta_{F} A_{\mu_{1} \ldots \mu_{8}}^{\alpha \beta} & =8 V_{+}^{(\alpha} V_{-}^{\beta)} \bar{\epsilon} \gamma_{\left[\mu_{1} \ldots \mu_{7}\right.} \psi_{\left.\mu_{8}\right]}+i V_{-}^{\alpha} V_{-}^{\beta} \bar{\epsilon}^{*} \gamma_{\mu_{1} \ldots \mu_{8}} \lambda+\text { c.c. },
\end{aligned}
$$

where all conventions are as in [7].

The commutators of two supersymmetry transformations on the fields and dual fields of type IIB were analysed in [12] at lowest order in the fermions. Given the transformations of eq. (2.3), together with the transformations of the scalars and the vielbein

$$
\begin{aligned}
\delta V_{+}^{\alpha} & =V_{-}^{\alpha} \bar{\epsilon}^{*} \lambda, \\
\delta e_{\mu}{ }^{a} & =i \bar{\epsilon} \gamma^{a} \psi_{\mu}+\text { c.c. }
\end{aligned}
$$

and the transformations of the fermions (without including cubic fermi terms)

$$
\begin{aligned}
\delta \psi_{\mu} & =D_{\mu} \epsilon+\frac{i}{480} F_{\mu \nu_{1} \ldots \nu_{4}} \gamma^{\nu_{1} \ldots \nu_{4}} \epsilon+\frac{1}{96} G^{\nu \rho \sigma} \gamma_{\mu \nu \rho \sigma} \epsilon^{*}-\frac{3}{32} G_{\mu \nu \rho} \gamma^{\nu \rho} \epsilon^{*}, \\
\delta \lambda & =i P_{\mu} \gamma^{\mu} \epsilon^{*}-\frac{i}{24} G_{\mu \nu \rho} \gamma^{\mu \nu \rho} \epsilon,
\end{aligned}
$$


where

$$
P_{\mu}=-\epsilon_{\alpha \beta} V_{+}^{\alpha} \partial_{\mu} V_{+}^{\beta}
$$

and

$$
G_{\mu \nu \rho}=-\epsilon_{\alpha \beta} V_{+}^{\alpha} F_{\mu \nu \rho}^{\beta},
$$

the commutators of two supersymmetry transformations on the bosons close on all the local symmetries of the theory, including the gauge transformations of eq. (2.1), provided that the duality relations

$$
\begin{aligned}
& F_{\mu_{1} \ldots \mu_{7}}^{\alpha}=-\frac{i}{3} \epsilon_{\mu_{1} \ldots \mu_{7} \nu_{1} \ldots \nu_{3}} V_{+}^{(\alpha} V_{-}^{\beta)} \epsilon_{\beta \gamma} F^{\gamma, \nu_{1} \ldots \nu_{3}}, \\
& F_{\mu_{1} \ldots \mu_{9}}^{\alpha \beta}=i \epsilon_{\mu_{1} \ldots \mu_{9}}{ }^{\sigma}\left[V_{+}^{\alpha} V_{+}^{\beta} P_{\sigma}^{*}-V_{-}^{\alpha} V_{-}^{\beta} P_{\sigma}\right]
\end{aligned}
$$

hold, together with the self-duality condition for the 5-form field-strength. What will be crucial in the following are the expressions for the gauge parameters of the gauge transformations resulting from the commutators of two supersymmetry transformations that are purely fermi bilinears, that are

$$
\begin{aligned}
\Lambda_{\mu}^{\alpha} & =-2 i V_{-}^{\alpha} \bar{\epsilon}_{2}^{*} \gamma_{\mu} \epsilon_{1}+\text { c.c. } \\
\Lambda_{\mu \nu \rho} & =-\frac{1}{4} \bar{\epsilon}_{2} \gamma_{\mu \nu \rho} \epsilon_{1}+\text { c.c. } \\
\Lambda_{\mu_{1} \ldots \mu_{5}}^{\alpha} & =-2 V_{-}^{\alpha} \bar{\epsilon}_{2}^{*} \gamma_{\mu_{1} \ldots \mu_{5}} \epsilon_{1}+\text { c.c. }, \\
\Lambda_{\mu_{1} \ldots \mu_{7}}^{\alpha \beta} & =-V_{+}^{(\alpha} V_{-}^{\beta)} \bar{\epsilon}_{2} \gamma_{\mu_{1} \ldots \mu_{7}} \epsilon_{1}+\text { c.c. }
\end{aligned}
$$

In [7] the closure of the supersymmetry algebra on the scalars, the vielbein, the 2-forms and the 4-form, as well as on the fermions, was obtained at all orders in the fermions. Given that the supersymmetry algebra closes on-shell, this analysis was used to derive the field equations requiring the closure of the algebra on the fermi fields. Here we want to perform a similar analysis for all the bosonic fields and their duals. For simplicity we will only consider terms that are quadratic in the gravitino, that is we will ignore all the higher order fermi terms containing the spinor $\lambda$. The advantage of this is that the modification of the supersymmetry transformations of eq. (2.6) and of the duality relations of eq. (2.9) are all determined by supercovariance as far as these terms are concerned. As it turns out, this analysis is sufficient to determine all the 10-forms that are compatible with supersymmetry, as will be shown in the next subsection.

The expressions for the supercovariant spin connection and field strengths (only considering terms quadratic in the gravitino) are

$$
\begin{aligned}
\hat{\omega}_{\mu a b} & =\omega_{\mu a b}+i e^{\nu}{ }_{a} e^{\rho}{ }_{b}\left[\bar{\psi}_{\mu} \gamma_{[\nu} \psi_{\rho]}+\bar{\psi}_{[\nu} \gamma_{\rho]} \psi_{\mu}+\bar{\psi}_{[\nu} \gamma_{|\mu|} \psi_{\rho]}\right] \\
\hat{F}_{\mu_{1} \ldots \mu_{3}}^{\alpha} & =F_{\mu_{1} \ldots \mu_{3}}^{\alpha}+\left[-6 i V_{-}^{\alpha} \bar{\psi}_{\left[\mu_{1}\right.}^{*} \gamma_{\mu_{2}} \psi_{\left.\mu_{3}\right]}+\text { c.c. }\right] \\
\hat{F}_{\mu_{1} \ldots \mu_{5}} & =F_{\mu_{1} \ldots \mu_{5}}-5 \bar{\psi}_{\left[\mu_{1}\right.} \gamma_{\mu_{2} \ldots \mu_{4}} \psi_{\left.\mu_{5}\right]}, \\
\hat{F}_{\mu_{1} \ldots \mu_{7}}^{\alpha} & =F_{\mu_{1} \ldots \mu_{7}}^{\alpha}+\left[-42 V_{-}^{\alpha} \bar{\psi}_{\left[\mu_{1}\right.}^{*} \gamma_{\mu_{2} \ldots \mu_{6}} \psi_{\left.\mu_{7}\right]}+\text { c.c. }\right] \\
\hat{F}_{\mu_{1} \ldots \mu_{9}}^{\alpha \beta} & =F_{\mu_{1} \ldots \mu_{9}}^{\alpha \beta}-72 V_{+}^{(\alpha} V_{-}^{\beta)} \bar{\psi}_{\left[\mu_{1}\right.} \gamma_{\mu_{2} \ldots \mu_{8}} \psi_{\left.\mu_{9}\right]} .
\end{aligned}
$$


The terms of the form $\epsilon^{2} \psi^{2}$ resulting in the commutators of two supersymmetry transformations on the form fields have two sources. The first are the terms, that we schematically write as $\left[\delta_{F}, \delta_{F}\right] A$, resulting from considering only the purely fermionic term in the supersymmetry variation of the form field, that is only the first term on the right hand side of each line of eq. (2.3). The resulting $\epsilon^{2} \psi^{2}$ terms can be immediately read by simply substituting the supercovariant quantities of eq. (2.11) to the bosonic result. The second source comes from the purely fermionic variation of the form fields in the $A \delta_{F} A$ terms in eq. (2.3), that is the terms $\delta_{F} A \delta_{F} A$. These can be immediately written using eq. (2.4), and in order to compare them to the previous ones one has to perform some Fierz rearrangements, using the Fierz identity

$$
\xi \bar{\chi}=-\frac{1}{16} \gamma_{\mu}\left(\bar{\chi} \gamma^{\mu} \xi\right)+\frac{1}{96} \gamma_{\mu \nu \rho}\left(\bar{\chi} \gamma^{\mu \nu \rho} \xi\right)-\frac{1}{3840} \gamma_{\mu \nu \rho \sigma \tau}\left(\bar{\chi} \gamma^{\mu \nu \rho \sigma \tau} \xi\right)
$$

Here $\chi$ and $\psi$ are two generic ten-dimensional spinors of the same chirality.

The final result is that the commutator of two supersymmetry transformations on the bosons generates a supersymmetry transformation of parameter

$$
\zeta=-\xi^{\mu} \psi_{\mu}
$$

where

$$
\xi_{\mu}=i \bar{\epsilon}_{2} \gamma_{\mu} \epsilon_{1}+\text { c.c. . }
$$

This is the supersymmetry parameter of [7] as far as the gravitino terms are concerned.

\section{$2.2 \quad$ Ten-form potentials}

We now want to extend this analysis to the 10-forms. In [12] it was shown that the supersymmetry algebra closes at lowest order in the fermions of a quadruplet and a doublet of 10-forms whose supersymmetry transformations are

$$
\begin{aligned}
\delta A_{\mu_{1} \ldots \mu_{10}}^{\alpha \beta \gamma}= & -\frac{20}{3} V_{+}^{(\alpha} V_{-}^{\beta} V_{-}^{\gamma)} \bar{\epsilon}^{*} \gamma_{\left[\mu_{1} \ldots \mu_{9}\right.} \psi_{\left.\mu_{10}\right]}-i V_{+}^{(\alpha} V_{-}^{\beta} V_{-}^{\gamma)} \bar{\epsilon} \gamma_{\mu_{1} \ldots \mu_{10}} \lambda+\text { c.c. } \\
& -12 A_{\left[\mu_{1} \ldots \mu_{8}\right.}^{(\alpha \beta} \delta_{F} A_{\left.\mu_{9} \mu_{10}\right]}^{\gamma)}+3 A_{\left[\mu_{1} \mu_{2}\right.}^{(\alpha} \delta_{F} A_{\left.\mu_{3} \ldots \mu_{10}\right]}^{\beta \gamma)} \\
\delta A_{\mu_{1} \ldots \mu_{10}}^{\alpha}= & 20 i V_{-}^{\alpha} \bar{\epsilon}^{*} \gamma_{\left[\mu_{1} \ldots \mu_{9}\right.} \psi_{\left.\mu_{10}\right]}+V_{-}^{\alpha} \bar{\epsilon} \gamma_{\mu_{1} \ldots \mu_{10}} \lambda+\text { c.c. }
\end{aligned}
$$

where the quadruplet has a non-trivial gauge transformation

$$
\delta A_{\mu_{1} \ldots \mu_{10}}^{\alpha \beta \gamma}=10 \partial_{\left[\mu_{1}\right.} \Lambda_{\left.\mu_{2} \ldots \mu_{10}\right]}^{(\alpha \beta \gamma)}-\frac{2}{3} F_{\left[\mu_{1} \ldots \mu_{9}\right.}^{(\alpha \beta} \Lambda_{\left.\mu_{10}\right]}^{\gamma)}+32 F_{\left[\mu_{1} \ldots \mu_{3}\right.}^{(\alpha} \Lambda_{\left.\mu_{4} \ldots \mu_{10}\right]}^{\beta \gamma \gamma}
$$

while the gauge transformation of the doublet is trivial:

$$
\delta A_{\mu_{1} \ldots \mu_{10}}^{\alpha}=10 \partial_{\left[\mu_{1}\right.} \Lambda_{\left.\mu_{2} \ldots \mu_{10}\right]}^{\alpha} .
$$

It turns out that there is an additional doublet of 10 -forms $\tilde{A}_{\mu_{1} \ldots \mu_{10}}$ on which the supersymmetry algebra closes at lowest order in the fermions. The supersymmetry transformation of this additional 10 -form is

$$
\begin{aligned}
\delta \tilde{A}_{10}^{\alpha}= & -V_{-}^{\alpha} \bar{\epsilon} \gamma_{\mu_{1} \ldots \mu_{10}} \lambda+\text { c.c. }+9 i \epsilon_{\beta \gamma} A_{\left[\mu_{1} \mu_{2}\right.}^{\beta} \delta_{F} A_{\left.\mu_{3} \ldots \mu_{10}\right]}^{\gamma \alpha}+252 A_{\left[\mu_{1} \ldots \mu_{4}\right.} \delta_{F} A_{\left.\mu_{5} \ldots \mu_{10}\right]}^{\alpha} \\
& -378 A_{\left[\mu_{1} \ldots \mu_{6}\right.}^{\alpha} \delta_{F} A_{\left.\mu_{7} \ldots \mu_{10}\right]}+36 i \epsilon_{\beta \gamma} A_{\left[\mu_{1} \ldots \mu_{8}\right.}^{\alpha \beta} \delta_{F} A_{\left.\mu_{9} \mu_{10}\right]}^{\gamma}
\end{aligned}
$$


while its gauge transformation is

$$
\begin{aligned}
\delta \tilde{A}_{\mu_{1} \ldots \mu_{10}}^{\alpha}= & 10 \partial_{\left[\mu_{1}\right.} \Lambda_{\left.\mu_{2} \ldots \mu_{10}\right]}+2 i \epsilon_{\beta \gamma} \Lambda_{\left[\mu_{1}\right.}^{\beta} F_{\left.\mu_{2} \ldots \mu_{10}\right]}^{\gamma \alpha}+144 \Lambda_{\left[\mu_{1} \ldots \mu_{3}\right.} F_{\left.\mu_{4} \ldots \mu_{10}\right]}^{\alpha} \\
& -\frac{2268}{5} \Lambda_{\left[\mu_{1} \ldots \mu_{5}\right.}^{\alpha} F_{\left.\mu_{6} \ldots \mu_{10}\right]}+96 i \epsilon_{\beta \gamma} F_{\left[\mu_{1} \ldots \mu_{3}\right.}^{\beta} \Lambda_{\left.\mu_{4} \ldots \mu_{10}\right]}^{\gamma \alpha} .
\end{aligned}
$$

In order to prove that the commutator of two supersymmetry transformations of eq. (2.18) closes on the gauge transformations of eq. (2.19) one makes use of the crucial identities

$$
\begin{aligned}
F_{\left[\mu_{1} \ldots \mu_{5}\right.} \Lambda_{\left.\mu_{6} \ldots \mu_{10}\right]}^{\alpha} & =0 \\
i \epsilon_{\beta \gamma} \Lambda_{\left[\mu_{1} \ldots \mu_{7}\right.}^{\alpha \beta} F_{\left.\mu_{8} \ldots \mu_{10}\right]}^{\gamma} & =-2 \Lambda_{\left[\mu_{1} \ldots \mu_{3}\right.} F_{\left.\mu_{4} \ldots \mu_{10}\right]}^{\alpha}, \\
2 i V_{-}^{\alpha} P_{\left[\mu_{1}\right.} \bar{\epsilon}_{2} \gamma_{\left.\mu_{2} \ldots \mu_{10}\right]} \epsilon_{1}^{*}+2 i V_{+}^{\alpha} P_{\left[\mu_{1}\right.}^{*} \bar{\epsilon}_{2}^{*} \gamma_{\left.\mu_{2} \ldots \mu_{10}\right]} \epsilon_{1} & =i \epsilon_{\beta \gamma} F_{\left[\mu_{1} \ldots \mu_{9}\right.}^{\alpha \beta} \Lambda_{\left.\mu_{10}\right]}^{\gamma},
\end{aligned}
$$

which are a consequence of the duality relations of eq. (2.9) and of the properties of the gamma matrices in ten dimensions. The reason why this additional doublet of 10 -forms was missed in [12] is because these identities were not used in those calculations. Of course, the supersymmetry algebra closes at lowest order in the fermions on any linear combinations of the trivial and the non-trivial doublet, and combining the non-trivial doublet with the trivial one does not change the form of the gauge transformations of eq. (2.19).

We now show that the non-trivial IIB doublet of 10 -forms is precisely the one predicted by $E_{11} \cdot{ }^{2}$ The $E_{11}$ analysis of the generators that is relevant for the IIB theory was performed originally in [15], while all the form generators were classified in [14]. The algebra involving all the form generators associated to the propagating form fields and the quadruplet of 10-form generators was derived in [16], where it was also shown that the symmetry of the group element exactly reproduces the gauge transformations of the corresponding fields as obtained in [12]. Including also the doublet of 10 -form generators this algebra is

$$
\begin{aligned}
{\left[R_{\alpha}^{\mu_{1} \mu_{2}}, R_{\beta}^{\mu_{3} \mu_{4}}\right] } & =i \epsilon_{\alpha \beta} R^{\mu_{1} \ldots \mu_{4}} & {\left[R_{\alpha}^{\mu_{1} \mu_{2}}, R^{\mu_{3} \ldots \mu_{6}}\right] } & =R_{\alpha}^{\mu_{1} \ldots \mu_{6}} \quad\left[R_{\alpha}^{\mu_{1} \mu_{2}}, R_{\beta}^{\mu_{3} \ldots \mu_{8}}\right]=R_{\alpha \beta}^{\mu_{1} \ldots \mu_{8}} \\
{\left[R^{\mu_{1} \ldots \mu_{4}}, R_{\alpha}^{\mu_{5} \ldots \mu_{10}}\right] } & =R_{\alpha}^{\mu_{1} \ldots \mu_{10}} & {\left[R_{\alpha}^{\mu_{1} \mu_{2}}, R_{\beta \gamma}^{\mu_{3} \ldots \mu_{10}}\right] } & =R_{\alpha \beta \gamma}^{\mu_{1} \ldots \mu_{10}}+\frac{2}{3} i \epsilon_{\alpha(\beta} R_{\gamma)}^{\mu_{1} \ldots \mu_{10}}
\end{aligned}
$$

with all the other commutators vanishing. One then considers the group element

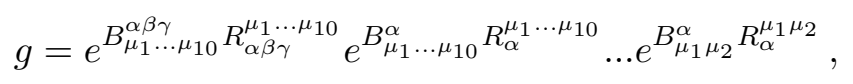

where the $B$ 's are the fields associated to each generator. Requiring symmetry under global transformations of the form $g \rightarrow g_{0} g$ gives the global transformations of the fields, and in particular for the fields up to the 10-forms one gets

$$
\begin{aligned}
\delta B_{\mu_{1} \mu_{2}}^{\alpha} & =a_{\mu_{1} \mu_{2}}^{\alpha}, \\
\delta B_{\mu_{1} \ldots \mu_{4}} & =a_{\mu_{1} \ldots \mu_{4}}+\frac{i}{2} \epsilon_{\alpha \beta} a_{\left[\mu_{1} \mu_{2}\right.}^{\alpha} B_{\left.\mu_{3} \mu_{4}\right]}^{\beta}, \\
\delta B_{\mu_{1} \ldots \mu_{6}}^{\alpha} & =a_{\mu_{1} \ldots \mu_{6}}^{\alpha}+a_{\left[\mu_{1} \mu_{2}\right.}^{\alpha} B_{\left.\mu_{3} \ldots \mu_{6}\right]}+\frac{i}{6} \epsilon_{\beta \gamma} a_{\left[\mu_{1} \mu_{2}\right.}^{\beta} B_{\mu_{3} \mu_{4}}^{\gamma} B_{\left.\mu_{5} \mu_{6}\right]}^{\alpha}, \\
\delta B_{\mu_{1} \ldots \mu_{8}}^{\alpha \beta} & =a_{\mu_{1} \ldots \mu_{8}}^{\alpha \beta}+a_{\left[\mu_{1} \mu_{2}\right.}^{(\alpha} B_{\left.\mu_{3} \ldots \mu_{8}\right]}^{\beta)}+\frac{i}{24} \epsilon_{\gamma \delta} a_{\left[\mu_{1} \mu_{2}\right.}^{\gamma} B_{\mu_{3} \mu_{4}}^{\delta} B_{\mu_{5} \mu_{6}}^{\alpha} B_{\left.\mu_{7} \mu_{8}\right]}^{\beta} .
\end{aligned}
$$

\footnotetext{
${ }^{2}$ We ignore here the ambiguity related to the fact that one can always add a trivial IIB doublet (times a constant) to a non-trivial IIB doublet. The same applies to the IIA case.
} 
One then recovers the gauge transformations of the fields by promoting the constant shifts to gauge transformations:

$$
a_{\mu_{1} \ldots \mu_{n}}=n \partial_{\left[\mu_{1}\right.} \Sigma_{\left.\mu_{2} \ldots \mu_{n}\right]} .
$$

The algebraic construction that in general leads to the gauge transformations starting from the global $E_{11}$ transformations was derived in [17]. One can show that after field redefinitions and redefinitions of the gauge parameters, the transformations of eq. (2.23) coincide with those of eq. (2.1). Similarly, one can determine from $E_{11}$ the transformation of the 10-form doublet $B_{\mu_{1} \ldots \mu_{10}}^{\alpha}$. After reinterpreting the global shifts as gauge transformations as in eq. (2.24) one obtains

$$
\begin{aligned}
\delta B_{\mu_{1} \ldots \mu_{10}}^{\alpha}= & 10 \partial_{\left[\mu_{1}\right.} \Sigma_{\left.\mu_{2} \ldots \mu_{10}\right]}^{\alpha}+4 \partial_{\left[\mu_{1}\right.} \Sigma_{\mu_{2} \ldots \mu_{4}} B_{\left.\mu_{5} \ldots \mu_{10}\right]}^{\alpha}+\frac{4}{3} i \epsilon_{\beta \gamma} \partial_{\left[\mu_{1}\right.} \Sigma_{\mu_{2}}^{\beta} B_{\left.\mu_{3} \ldots \mu_{10}\right]}^{\gamma \alpha} \\
& +\partial_{\left[\mu_{1}\right.} \Sigma_{\mu_{2}}^{\alpha} B_{\mu_{3} \ldots \mu_{6}} B_{\left.\mu_{7} \ldots \mu_{10}\right]}+\frac{i}{3} \epsilon_{\beta \gamma} \partial_{\left[\mu_{1}\right.} \Sigma_{\mu_{2}}^{\beta} B_{\mu_{3} \mu_{4}}^{\gamma} B_{\mu_{5} \mu_{6}}^{\alpha} B_{\left.\mu_{7} \ldots \mu_{10}\right]} .
\end{aligned}
$$

After field redefinitions and redefinitions of the gauge parameters one can show that this gauge transformation coincides with the one in eq. (2.19). This thus shows that the new doublet of 10 -forms $\tilde{A}_{\mu_{1} \ldots \mu_{10}}^{\alpha}$ is the one predicted by $E_{11}$.

We now consider the commutator of two supersymmetry transformations on the 10forms of IIB supergravity, only considering the terms that do not contain the spinor $\lambda$. For the case of the quadruplet, the result is exactly as for the lower rank forms discussed in the previous subsection, and the commutator of two supersymmetry transformations generates a supersymmetry transformation with parameter as given in eq. (2.13). The picture changes when one considers the two doublets. One can immediately show using the ten-dimensional Fierz identities of eq. (2.12) that the supersymmetry algebra does not close on both the trivial doublet transforming as in eq. (2.15) and on the non-trivial doublet transforming as in eq. (2.18). Only for a particular combination of these two fields one obtains closure, and the result is that the only doublet of 10-forms compatible with supersymmetry is

$$
\tilde{A}_{\mu_{1} \ldots \mu_{10}}^{\alpha}-\frac{23}{16} A_{\mu_{1} \ldots \mu_{10}}^{\alpha} .
$$

This analysis thus produces the intriguing result that for top-forms the closure of the supersymmetry algebra at lowest order in the fermions does not in general guarantee actual closure at the full level. As we will see in the next section, the same result applies to the IIA case.

\section{$2.3 \quad$ IIB superspace}

The superspace version of this story is of course equivalent to the component one just described, but the organisation of the calculation differs somewhat. In the superspace approach it is preferable to work with tensorial quantities, rather than gauge potentials, so that supersymmetry as well as gauge invariance is manifest at every step. On the other hand, the introduction of field strengths in the odd (spinorial) directions as well as the even (spacetime) ones, and the fact that each field is now a superfield, means that constraints must be imposed in order to get rid of the non-physical fields. The procedure is therefore to impose these on the various field strengths and then to check that they are consistent 
by examining the Bianchi identities. It is actually rather easy to find the constraints when one knows the field content of the theory simply by using dimensional analysis. A feature of this approach is that we can examine the field strength even for a ten-form potential because an eleven-form need not vanish in the superspace context due to the fact that the odd basis differential forms are commutative.

For the IIB case, the full theory was written down in terms of the usual physical fields in [8] and then extended to include the dual forms in [18]; later, in [13], all of these plus the eleven-form field strengths were included. The full list of Bianchi identities and the nonvanishing components of all of the forms can be found there; here we shall just re-examine the eleven-forms. There is a quadruplet $F_{11}^{\alpha \beta \gamma}$ which obeys the Bianchi

$$
d F_{11}^{\alpha \beta \gamma}=F_{3}^{(\alpha} F_{9}^{\beta \gamma)},
$$

and a doublet, $F_{11}^{\alpha}$, for which the Bianchi identity is

$$
d F_{11}^{\alpha}=\frac{4}{23}\left(\epsilon_{\beta \gamma} F_{3}^{\beta} F_{9}^{\gamma \alpha}-\frac{3}{4} F_{5} F_{7}^{\alpha}\right) .
$$

Any $n$-form in superspace can be split up into a sum of $(p, q)$-forms, where $p(q)$ denotes the number of even (odd) indices and where $n=p+q{ }^{3}$ For an $n$-form field strength $F$, the top component, $F_{n, 0}$, has dimension one, so that the only other ones which can be non-zero are $F_{n-2,2}$ and $F_{n-1,1}$ which have dimensions zero and one-half respectively. In a $\mathrm{U}(1)$ frame (reached by means of the scalar field matrix $V$ acting on the $\mathrm{SL}(2, R)$ indices) the dimension-zero component will be a gamma-matrix times some internal invariant if appropriate, while the dimension one-half component will be proportional to the dilatino. It will be useful to think of the symmetric $p$-index gamma-matrices as $(p, 2)$ forms, written $\gamma_{p, 2}$, and the product of a gamma-matrix with the fermion as a $(p, 1)$-form, written $(\gamma \cdot \lambda)_{p, 1}$. For the eleven-forms the dimension-zero and one-half components are precisely of this type; the full details can be found in [13].

Now we ask if there can be a gauge-trivial doublet of eleven-forms, i.e. an $F_{11}^{\alpha}$ satisfying $d F_{11}^{\alpha}=0$. The first non-trivial component of this identity, at dimension zero, can be written

$$
t_{0} F_{9,2}^{\alpha}=0,
$$

where $t_{0}$ denotes an algebraic operation formed by contracting the even-vector index of the dimension-zero torsion, which is proportional to a gamma-matrix regarded as an evenvector-valued $(0,2)$-form, with one of the even indices of the form being operated on, and where all the remaining odd indices are symmetrised. It is quite easy to see that there is no non-trivial gamma-matrix identity that satisfies $(2.29)$, so that $F_{9,2}^{\alpha}=0$. But then this implies, using the dimension one-half Bianchi, that $F_{10,1}^{\alpha}$ is also zero, and so the whole of $F$ must vanish.

The component results can be recovered from superspace by observing that a supersymmetry transformation can be regarded as a super-diffeomorphism with an odd vector field whose leading component (in an odd coordinate expansion) is identified with the local

\footnotetext{
${ }^{3}$ Note that this splitting is invariant with respect to a class of preferred non-coordinate basis frames.
} 
supersymmetry parameter in spacetime. It is not difficult to show that the transformation of a $p$-form potential is given by the interior product of this vector field with the field strength $F_{p+1}$. The $\lambda$ terms in the variation come from $F_{p, 1}$ while the gravitino terms come from $F_{p, 2}$. The latter arises because one has to go from a preferred basis to a coordinate basis by means of the supervielbein, one component of which is the gravitino.

\section{The top-forms of IIA supergravity}

In this section we repeat the same analysis for the IIA case. In [11] the supersymmetry transformations for all the forms of the IIA theory were derived, and the closure of the supersymmetry algebra was checked at lowest order in the fermions. This analysis was performed also in the case of non-vanishing Romans mass, and apart from all the propagating forms, it was also done for the 9-form potential, whose field strength is dual to the Romans mass, and for a non-trivial 10-form and a trivial one. In this section we will reconsider the analysis of the 10-forms, and for simplicity we will consider the case of vanishing Romans mass. We will first review the analysis for all the forms up to the 10-forms. We will then show that an additional non-trivial 10-form can be included, while the closure of the supersymmetry algebra at all orders in the fermions selects two 10-forms out of the three that are a priori compatible with supersymmetry at lowest order. Finally, we will perform the same analysis in superspace.

\subsection{IIA supergravity}

We follow the notation of [9], which is the one also used in [11]. The supersymmetry transformations are thus expressed in the string frame, and we use the mostly plus signature, as opposed to the one used in the previous section. We denote with $C$ the RR fields and with $B$ the NS-NS fields. The RR fields are forms of odd rank, while the NS-NS fields are the 2 -form, the 6 -form and the 8 -form. With respect to ref. [11], we perform field redefinitions for the 6 -form and the 8 -form, so that their gauge transformations are in the abelian basis as is the case for all the other fields. The resulting gauge transformations are

$$
\begin{aligned}
\delta C_{\mu_{1} \ldots \mu_{2 n-1}}= & (2 n-1) \partial_{\left[\mu_{1}\right.} \Lambda_{\left.\mu_{2} \ldots \mu_{2 n-1}\right]}-\left(\begin{array}{c}
2 n-1 \\
3
\end{array}\right) H_{\left[\mu_{1} \ldots \mu_{3}\right.} \Lambda_{\left.\mu_{4} \ldots \mu_{2 n-1}\right]} \\
\delta B_{\mu_{1} \mu_{2}}= & 2 \partial_{\left[\mu_{1}\right.} \Sigma_{\left.\mu_{2}\right]}, \\
\delta B_{\mu_{1} \ldots \mu_{6}}= & 6 \partial_{\left[\mu_{1}\right.} \Sigma_{\left.\mu_{2} \ldots \mu_{6}\right]}-\frac{15}{2} G_{\left[\mu_{1} \mu_{2}\right.} \Lambda_{\left.\mu_{3} \ldots \mu_{6}\right]}+\frac{15}{2} G_{\left[\mu_{1} \ldots \mu_{4}\right.} \Lambda_{\left.\mu_{5} \mu_{6}\right]}-\frac{1}{6} G_{\mu_{1} \ldots \mu_{6}} \Lambda \\
\delta B_{\mu_{1} \ldots \mu_{8}}= & 8 \partial_{\left[\mu_{1}\right.} \Sigma_{\left.\mu_{2} \ldots \mu_{8}\right]}+21 G_{\left[\mu_{1} \mu_{2}\right.} \Lambda_{\left.\mu_{3} \ldots \mu_{8}\right]}-35 G_{\left[\mu_{1} \ldots \mu_{4}\right.} \Lambda_{\left.\mu_{5} \ldots \mu_{8}\right]}+7 G_{\left[\mu_{1} \ldots \mu_{6}\right.} \Lambda_{\left.\mu_{7} \mu_{8}\right]} \\
& +28 H_{\left[\mu_{1} \ldots \mu_{3}\right.} \Sigma_{\left.\mu_{4} \ldots \mu_{8}\right]}
\end{aligned}
$$

while the corresponding field strengths are

$$
\begin{aligned}
G_{\mu_{1} \ldots \mu_{2 n}}= & 2 n \partial_{\left[\mu_{1}\right.} C_{\left.\mu_{2} \ldots \mu_{2 n}\right]}-\left(\begin{array}{c}
2 n \\
3
\end{array}\right) H_{\left[\mu_{1} \ldots \mu_{3}\right.} C_{\left.\mu_{4} \ldots \mu_{2 n}\right]} \\
H_{\mu_{1} \ldots \mu_{3}}= & 3 \partial_{\left[\mu_{1}\right.} B_{\left.\mu_{2} \mu_{3}\right]}, \\
H_{\mu_{1} \ldots \mu_{7}}= & 7 \partial_{\left[\mu_{1}\right.} B_{\left.\mu_{2} \ldots \mu_{7}\right]}+\frac{21}{2} G_{\left[\mu_{1} \mu_{2}\right.} C_{\left.\mu_{3} \ldots \mu_{7}\right]}-\frac{35}{2} G_{\left[\mu_{1} \ldots \mu_{4}\right.} C_{\left.\mu_{5} \ldots \mu_{7}\right]}+\frac{7}{2} G_{\left[\mu_{1} \ldots \mu_{6}\right.} C_{\left.\mu_{7}\right]} \\
H_{\mu_{1} \ldots \mu_{9}}= & 9 \partial_{\left[\mu_{1}\right.} B_{\left.\mu_{2} \ldots \mu_{9}\right]}-27 G_{\left[\mu_{1} \mu_{2}\right.} C_{\left.\mu_{3} \ldots \mu_{9}\right]}+63 G_{\left[\mu_{1} \ldots \mu_{4}\right.} C_{\left.\mu_{5} \ldots \mu_{9}\right]}-21 G_{\left[\mu_{1} \ldots \mu_{6}\right.} C_{\left.\mu_{7} \ldots \mu_{9}\right]} \\
& +42 B_{\left[\mu_{1} \ldots \mu_{6}\right.} H_{\left.\mu_{7} \ldots \mu_{9}\right]} .
\end{aligned}
$$


As in the IIB case, in this basis the supersymmetry transformations have a particularly simple form, in which all terms are at most linear in the form fields. The result is

$$
\begin{aligned}
\delta C_{\mu_{1} \ldots \mu_{2 n-1}}= & \delta_{F} C_{\mu_{1} \ldots \mu_{2 n-1}}+\left(\begin{array}{c}
2 n-1 \\
2
\end{array}\right) C_{\left[\mu_{1} \ldots \mu_{2 n-3}\right.} \delta_{F} B_{\left.\mu_{2 n-2} \mu_{2 n-1}\right]}, \\
\delta B_{\mu_{1} \mu_{2}}= & \delta_{F} B_{\mu_{1} \mu_{2}}, \\
\delta B_{\mu_{1} \ldots \mu_{6}}= & \delta_{F} B_{\mu_{1} \ldots \mu_{6}}+3 C_{\left[\mu_{1} \ldots \mu_{5}\right.} \delta_{F} C_{\left.\mu_{6}\right]}-10 C_{\left[\mu_{1} \ldots \mu_{3}\right.} \delta_{F} C_{\left.\mu_{4} \ldots \mu_{6}\right]}+3 C_{\left[\mu_{1}\right.} \delta_{F} C_{\left.\mu_{2} \ldots \mu_{6}\right]}, \\
\delta B_{\mu_{1} \ldots \mu_{8}}= & \delta_{F} B_{\mu_{1} \ldots \mu_{8}}-6 C_{\left[\mu_{1} \ldots \mu_{7}\right.} \delta_{F} C_{\left.\mu_{8}\right]}+28 C_{\left[\mu_{1} \ldots \mu_{5}\right.} \delta_{F} C_{\left.\mu_{6} \ldots \mu_{8}\right]}-14 C_{\left[\mu_{1} \ldots \mu_{3}\right.} \delta_{F} C_{\left.\mu_{4} \ldots \mu_{8}\right]} \\
& -14 B_{\left[\mu_{1} \ldots \mu_{6}\right.} \delta_{F} B_{\left.\mu_{7} \mu_{8}\right]},
\end{aligned}
$$

where as in the previous section we denote with $\delta_{F}$ the part of the supersymmetry transformation that only involves fermi bilinears, that is

$$
\begin{aligned}
\delta_{F} C_{\mu_{1} \ldots \mu_{2 n-1}} & =-(2 n-1) \bar{\epsilon} \gamma_{\left[\mu_{1} \ldots \mu_{2 n-2}\right.} \gamma_{11}^{n} \psi_{\left.\mu_{2 n-1}\right]}+\frac{1}{2} \bar{\epsilon} \gamma_{11}^{n} \gamma_{\mu_{1} \ldots \mu_{2 n-1}} \lambda \\
\delta_{F} B_{\mu_{1} \mu_{2}} & =2 \bar{\epsilon} \gamma_{11} \gamma_{\left[\mu_{1}\right.} \psi_{\left.\mu_{2}\right]} \\
\delta_{F} B_{\mu_{1} \ldots \mu_{6}} & =6 e^{-2 \phi} \bar{\epsilon} \gamma_{\left[\mu_{1} \ldots \mu_{5}\right.} \psi_{\left.\mu_{6}\right]}-e^{-2 \phi_{\bar{\epsilon}}} \gamma_{\mu_{1} \ldots \mu_{6}} \lambda \\
\delta_{F} B_{\mu_{1} \ldots \mu_{8}} & =\frac{1}{2} e^{-2 \phi} \bar{\epsilon} \gamma_{\mu_{1} \ldots \mu_{8}} \gamma_{11} \lambda
\end{aligned}
$$

Given the supersymmetry transformations of the form fields of eq. (3.3), together with the supersymmetry transformations of the vielbein and the dilaton

$$
\begin{aligned}
\delta e_{\mu}{ }^{a} & =\bar{\epsilon} \gamma^{a} \psi_{\mu} \\
\delta \phi & =\frac{1}{2} \bar{\epsilon} \lambda
\end{aligned}
$$

as well as the supersymmetry transformations of the fermions at lowest order in the fermions,

$$
\begin{aligned}
\delta \psi_{\mu} & =D_{\mu} \epsilon+\frac{1}{8} H_{\mu \nu \rho} \Gamma^{\nu \rho} \Gamma_{11} \epsilon+\frac{1}{16} e^{\phi} G_{\nu \rho} \Gamma^{\nu \rho} \Gamma_{\mu} \Gamma_{11} \epsilon+\frac{1}{8 \cdot 4 !} e^{\phi} G_{\mu_{1} \ldots \mu_{4}} \Gamma^{\mu_{1} \ldots \mu_{4}} \Gamma_{\mu} \epsilon, \\
\delta \lambda & =\partial_{\mu} \phi \Gamma^{\mu} \epsilon-\frac{1}{12} H_{\mu \nu \rho} \Gamma_{11} \Gamma^{\mu \nu \rho} \epsilon+\frac{3}{8} e^{\phi} G_{\mu \nu} \Gamma_{11} \Gamma^{\mu \nu} \epsilon+\frac{1}{4 \cdot 4 !} e^{\phi} G_{\mu_{1} \ldots \mu_{4}} \Gamma^{\mu_{1} \ldots \mu_{4}} \epsilon,
\end{aligned}
$$

it was shown in [11] that the supersymmetry algebra closes at lowest order in the fermi fields, provided that the following duality relations hold:

$$
\begin{aligned}
G_{\mu_{1} \ldots \mu_{2 n}} & =(-1)^{n} \frac{1}{(10-2 n) !} \epsilon_{\mu_{1} \ldots \mu_{2 n}}{ }^{\mu_{2 n+1} \ldots \mu_{10}} G_{\mu_{2 n+1} \ldots \mu_{10}}, \\
H_{\mu_{1} \ldots \mu_{7}} & =\frac{1}{6} e^{-2 \phi} \epsilon_{\mu_{1} \ldots \mu \mu \mu \nu \rho} H^{\mu \nu \rho} \\
H_{\mu_{1} \ldots \mu_{9}} & =e^{-2 \phi} \epsilon_{\mu_{1} \ldots \mu_{9} \rho} \partial^{\rho} \phi .
\end{aligned}
$$

The fact that we are considering the massless theory in this paper implies in particular that $G_{10}$ vanishes as can be seen from the first equation. The closure of the supersymmetry algebra implies in particular that the commutator of two supersymmetry transformations produces the gauge transformations of eq. (3.1). What will be needed in the following is the explicit expression for the purely fermionic parts of the corresponding gauge parameters. These are

$$
\begin{aligned}
& \Lambda_{\mu_{1} \ldots \mu_{2 n}}=-e^{-\phi_{\epsilon_{2}}} \gamma_{\mu_{1} \ldots \mu_{2 n}} \gamma_{11}^{n+1} \epsilon_{1}, \\
& \Sigma_{\mu}=-\bar{\epsilon}_{2} \gamma_{11} \gamma_{\mu} \epsilon_{1},
\end{aligned}
$$

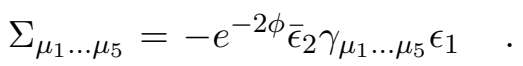


Note in particular that there is no purely fermionic part in the gauge parameter of the 8-form potential.

The analysis of [11] can be extended to include the quartic fermi terms. In particular, if one restricts one's attention to all terms that do not contain the spinor $\lambda$, then the modification of the supersymmetry transformations of the fermions in eq. (3.6) and of the duality relations of eq. (3.7) are fully determined by supercovariance. We thus replace in such equations the spin connection and the field strengths with the supercovariant expressions (again neglecting $\lambda$ contributions) ${ }^{4}$

$$
\begin{aligned}
\hat{\omega}_{\mu, a b} & =\omega_{\mu, a b}+\frac{1}{2} e_{a}^{\nu} e_{b}^{\rho}\left[\bar{\psi}_{\nu} \gamma_{\rho} \psi_{\mu}+\bar{\psi}_{\mu} \gamma_{\nu} \psi_{\rho}+\bar{\psi}_{\nu} \gamma_{\mu} \psi_{\rho}\right] \\
\hat{G}_{\mu_{1} \ldots \mu_{2 n}} & =G_{\mu_{1} \ldots \mu_{2 n}}+n(2 n-1) e^{-\phi} \bar{\psi}_{\left[\mu_{1}\right.} \gamma_{\mu_{2} \ldots \mu_{2 n-1}} \gamma_{11}^{n} \psi_{\left.\mu_{2 n}\right]}, \\
\hat{H}_{\mu_{1} \ldots \mu_{3}} & =H_{\mu_{1} \ldots \mu_{3}}-3 \bar{\psi}_{\left[\mu_{1}\right.} \gamma_{11} \gamma_{\mu_{2}} \psi_{\left.\mu_{3}\right]}, \\
\hat{H}_{\mu_{1} \ldots \mu_{7}} & =H_{\mu_{1} \ldots \mu_{7}}-21 \bar{\psi}_{\left[\mu_{1}\right.} \gamma_{\mu_{2} \ldots \mu_{6}} \psi_{\left.\mu_{7}\right]} .
\end{aligned}
$$

The calculation then proceeds exactly as in the IIB case discussed in the previous section. The terms of the form $\epsilon^{2} \psi^{2}$ resulting in the commutators of two supersymmetry transformations on the form fields are the terms resulting from considering only the purely fermionic term in the supersymmetry variation of the form field, that is only the first term on the right hand side of each line of eq. (3.3), and the terms coming from the purely fermionic variation of the form fields in eq. (3.3). The first can be immediately written by simply substituting the supercovariant expressions of eq. (3.9) to the bosonic result, while the latter are simply read from eq. (3.4). In order to compare the terms, we have to perform some Fierz rearrangements. Given that the IIA spinors are not chiral, we have to use the Fierz identity

$$
\xi \bar{\chi}=-\frac{1}{16}(\bar{\chi} \xi)+\frac{1}{32} \gamma_{\mu \nu}\left(\bar{\chi} \gamma^{\mu \nu} \xi\right)-\frac{1}{384} \gamma_{\mu \nu \rho \sigma}\left(\bar{\chi} \gamma^{\mu \nu \rho \sigma} \xi\right)
$$

where $\chi$ and $\xi$ are generic ten-dimensional spinors with opposite chirality, together with the Fierz identity of eq. (2.12), which applies when the chirality of the two spinors is the same. One can then show that the commutator of two supersymmetry transformations produces a supersymmetry transformation with parameter

$$
\zeta=-\xi^{\mu} \psi_{\mu}
$$

where we denote with $\xi_{\mu}$ the parameter of general coordinate transformations

$$
\xi_{\mu}=\bar{\epsilon}_{2} \gamma_{\mu} \epsilon_{1}
$$

We now want to repeat this analysis for the 10-forms.

\footnotetext{
${ }^{4}$ Note that the super-covariant curvature $\hat{H}_{\mu_{1} \ldots \mu_{9}}$ does not contain any gravitino squared terms.
} 


\section{$3.2 \quad$ Ten-form potentials}

Using the duality relations of eq. (3.7) and the expressions of eq. (3.8), one derives the following crucial identities:

$$
\begin{aligned}
\Lambda_{\left[\mu_{1} \mu_{2}\right.} G_{\left.\mu_{3} \ldots \mu_{10}\right]} & =\Lambda_{\left[\mu_{1} \ldots \mu_{8}\right.} G_{\left.\mu_{9} \mu_{10}\right]}, \\
\Lambda_{\left[\mu_{1} \ldots \mu_{4}\right.} G_{\left.\mu_{5} \ldots \mu_{10}\right]} & =\Lambda_{\left[\mu_{1} \ldots \mu_{6}\right.} G_{\left.\mu_{7} \ldots \mu_{10}\right]}, \\
\Sigma_{\left[\mu_{1}\right.} H_{\left.\mu_{2} \ldots \mu_{10}\right]} & =\partial_{\left[\mu_{1}\right.} \phi e^{-2 \phi_{\bar{\epsilon}_{2}} \gamma_{\left.\mu_{2} \ldots \mu_{10}\right]}} \epsilon_{1} .
\end{aligned}
$$

Using these identities one can show that the supersymmetry algebra at lowest order in the fermions closes on two independent 10-forms transforming non-trivially under gauge transformations. The gauge transformations of these 10-forms can be written in the abelian base exactly as for the forms of lower rank. They read

$$
\begin{aligned}
\delta B_{\mu_{1} \ldots \mu_{10}}= & 10 \partial_{\left[\mu_{1}\right.} \Sigma_{\left.\mu_{2} \ldots \mu_{10}\right]}+\frac{135}{2} G_{\left[\mu_{1} \mu_{2}\right.} \Lambda_{\left.\mu_{3} \ldots \mu_{10}\right]}-210 G_{\left[\mu_{1} \ldots \mu_{6}\right.} \Lambda_{\left.\mu_{7} \ldots \mu_{10}\right]} \\
& +\frac{135}{2} G_{\left[\mu_{1} \ldots \mu_{8}\right.} \Lambda_{\left.\mu_{9} \mu_{10}\right]}-\frac{3}{2} G_{\mu_{1} \ldots \mu_{10}} \Lambda-240 H_{\left[\mu_{1} \ldots \mu_{3}\right.} \Sigma_{\left.\mu_{4} \ldots \mu_{10}\right]}, \\
\delta \tilde{B}_{\mu_{1} \ldots \mu_{10}}= & \left.10 \partial_{\left[\mu_{1}\right.} \tilde{\Sigma}_{\left.\mu_{2} \ldots \mu_{10}\right]}+315 G_{\left[\mu_{1} \ldots \mu_{4}\right.} \Lambda_{\left.\mu_{5} \ldots \mu_{10}\right]}-525 G_{\left[\mu_{1} \ldots \mu_{6}\right.} \Lambda_{\left.\mu_{7} \ldots \mu_{10}\right]}\right] \\
& +135 G_{\left[\mu_{1} \ldots \mu_{8}\right.} \Lambda_{\left.\mu_{9} \mu_{10}\right]}-3 G_{\mu_{1} \ldots \mu_{10}} \Lambda-240 H_{\left[\mu_{1} \ldots \mu_{3}\right.} \Sigma_{\left.\mu_{4} \ldots \mu_{10}\right]},
\end{aligned}
$$

while the supersymmetry transformations in this base are

$$
\begin{aligned}
& \delta B_{\mu_{1} \ldots \mu_{10}}=\bar{\epsilon} \gamma_{\mu_{1} \ldots \mu_{10}} \lambda-15 C_{\left[\mu_{1} \ldots \mu_{9}\right.} \delta_{F} C_{\left.\mu_{10}\right]}+252 C_{\left[\mu_{1} \ldots \mu_{5}\right.} \delta_{F} C_{\left.\mu_{6} \ldots \mu_{10}\right]} \\
& \\
&-180 C_{\left[\mu_{1} \ldots \mu_{3}\right.} \delta_{F} C_{\left.\mu_{4} \ldots \mu_{10}\right]}+15 C_{\left[\mu_{1}\right.} \delta_{F} C_{\left.\mu_{2} \ldots \mu_{10}\right]}+90 B_{\left[\mu_{1} \ldots \mu_{8}\right.} \delta_{F} B_{\left.\mu_{9} \mu_{10}\right]} \\
& \delta \tilde{B}_{\mu_{1} \ldots \mu_{10}}=\bar{\epsilon} \gamma_{\mu_{1} \ldots \mu_{10}} \lambda-180 C_{\left[\mu_{1} \ldots \mu_{7}\right.} \delta_{F} C_{\left.\mu_{8} \ldots \mu_{10}\right]}+630 C_{\left[\mu_{1} \ldots \mu_{5}\right.} \delta_{F} C_{\left.\mu_{6} \ldots \mu_{10}\right]} \\
&-360 C_{\left[\mu_{1} \ldots \mu_{3}\right.} \delta_{F} C_{\left.\mu_{4} \ldots \mu_{10}\right]}+30 C_{\left[\mu_{1}\right.} \delta_{F} C_{\left.\mu_{2} \ldots \mu_{10}\right]}+90 B_{\left[\mu_{1} \ldots \mu_{8}\right.} \delta_{F} B_{\left.\mu_{9} \mu_{10}\right]}
\end{aligned}
$$

This analysis thus completes and corrects the one of ref. [11], were only one combination of these two 10-forms was found because the identities of eq. (3.13) were basically missed. As shown in [9], the supersymmetry algebra at lowest order in the fermions also closes on the trivial 10 -form $D_{10}$, whose supersymmetry transformations is

$$
\delta D_{\mu_{1} \ldots \mu_{10}}=e^{-2 \phi}\left[-10 \bar{\epsilon} \gamma_{\left[\mu_{1} \ldots \mu_{9}\right.} \psi_{\left.\mu_{10}\right]}+\bar{\epsilon} \gamma_{\mu_{1} \ldots \mu_{10}} \lambda\right],
$$

and whose gauge transformation is simply $\delta D_{10}=d \Lambda_{9}$.

Before analysing the supersymmetry algebra on these 10-forms at quartic order in the fermions, we want to show that the two non-trivial 10-forms are precisely those predicted by $E_{11}$. The way to obtain the IIA theory from $E_{11}$ was discussed originally in [19]. The analysis of all the commutation relations involving the generators up to the 10-form generators, as well as the computation of all the gauge transformations and the field strengths for all the fields up to the 10-forms, was performed in [17]. We refer to eq. (5.1) of that paper for the algebraic conventions. We add to those commutators the ones that produce the 10-form generators, which are

$$
\begin{aligned}
{\left[R^{\mu_{1} \mu_{2}}, R^{\mu_{3} \ldots \mu_{10}}\right] } & =R^{\mu_{1} \ldots \mu_{10}} & {\left[R^{\mu_{1} \ldots \mu_{3}}, R^{\mu_{4} \ldots \mu_{10}}\right] } & =R^{\mu_{1} \ldots \mu_{10}}+2 \tilde{R}^{\mu_{1} \ldots \mu_{10}} \\
{\left[R^{\mu_{1} \ldots \mu_{5}}, R^{\mu_{6} \ldots \mu_{10}}\right] } & =\tilde{R}^{\mu_{1} \ldots \mu_{10}} & {\left[R^{\mu_{1}}, R^{\mu_{2} \ldots \mu_{10}}\right] } & =4 R^{\mu_{1} \ldots \mu_{10}}+2 \tilde{R}^{\mu_{1} \ldots \mu_{10}}
\end{aligned}
$$


where $R^{\mu_{1} \ldots \mu_{10}}$ and $\tilde{R}^{\mu_{1} \ldots \mu_{10}}$ are the two independent 10 -form generators. If one then considers the group element

$$
g=e^{A_{\mu_{1} \ldots \mu_{10}} R^{\mu_{1} \ldots \mu_{10}}} e^{\tilde{A}_{\mu_{1} \ldots \mu_{10}} \tilde{R}^{\mu_{1} \ldots \mu_{10}}} e^{A_{\mu_{1} \ldots \mu_{9}} R^{\mu_{1} \ldots \mu_{9}}} \ldots e^{A_{\mu} R^{\mu}}
$$

where the $A$ 's are the fields associated to each generator, and requires symmetry under global transformations of the form $g \rightarrow g_{0} g$, one obtains the global transformations of the fields. In particular for the 10 -forms one gets

$$
\begin{aligned}
\delta A_{\mu_{1} \ldots \mu_{10}}= & a_{\mu_{1} \ldots \mu_{10}}+a_{\left[\mu_{1} \ldots \mu_{3}\right.} A_{\left.\mu_{4} \ldots \mu_{10}\right]}+a_{\left[\mu_{1} \mu_{2}\right.} A_{\left.\mu_{3} \ldots \mu_{10}\right]}+4 a_{\left[\mu_{1}\right.} A_{\left.\mu_{2} \ldots \mu_{10}\right]} \\
& -\frac{1}{3} A_{\left[\mu_{1} \ldots \mu_{3}\right.} A_{\mu_{4} \mu_{5}} A_{\mu_{6} \mu_{7}} A_{\mu_{8} \mu_{9}} a_{\left.\mu_{10}\right]} \\
\delta \tilde{A}_{\mu_{1} \ldots \mu_{10}}= & \tilde{a}_{\mu_{1} \ldots \mu_{10}}+\frac{1}{2} a_{\left[\mu_{1} \ldots \mu_{5}\right.} A_{\left.\mu_{6} \ldots \mu_{10}\right]}+2 a_{\left[\mu_{1} \ldots \mu_{3}\right.} A_{\left.\mu_{4} \ldots \mu_{10}\right]}+a_{\left[\mu_{1} \mu_{2}\right.} A_{\mu_{3} \ldots \mu_{5}} A_{\left.\mu_{6} \ldots \mu_{10}\right]} \\
& +2 a_{\left[\mu_{1}\right.} A_{\left.\mu_{2} \ldots \mu_{10}\right]}+\frac{2}{3} a_{\left[\mu_{1}\right.} A_{\mu_{2} \ldots \mu_{4}} A_{\mu_{5} \mu_{6}} A_{\mu_{7} \mu_{8}} A_{\left.\mu_{9} \mu_{10}\right]}+\frac{1}{2} a_{\left[\mu_{1}\right.} A_{\mu_{2} \ldots \mu_{6}} A_{\mu_{7} \mu_{8}} A_{\left.\mu_{9} \mu_{10}\right]} .
\end{aligned}
$$

One then recovers the gauge transformations of the fields by promoting the constant shifts to gauge transformations in a way analogous to eq. (2.24). One can show that after field redefinitions and redefinitions of the gauge parameters, the transformations of eq. (3.20) coincide with two linear combinations of the gauge transformations of eq. (3.14), which shows that the two non-trivial ten-form we found are exactly those predicted by $E_{11}$.

We now repeat for the IIA 10 -forms the same analysis that was performed for the doublets of 10-forms of the IIB theory. We consider the commutator of two supersymmetry transformations on the IIA 10-forms considering all the fermionic terms that are quadratic in the gravitino. One can immediately see using the ten-dimensional Fierz identities of eqs. (2.12) and (3.10) that the supersymmetry algebra does not close on any of the 10forms transforming under supersymmetry as in eqs. (3.15) and (3.16). One only obtains closure by considering a particular combination of each of the non-trivial 10-forms with the trivial one. The result is that the only two 10-forms compatible with supersymmetry are

$$
\begin{aligned}
& B_{\mu_{1} \ldots \mu_{10}}-14 D_{\mu_{1} \ldots \mu_{10}}, \\
& \tilde{B}_{\mu_{1} \ldots \mu_{10}}-38 D_{\mu_{1} \ldots \mu_{10}} .
\end{aligned}
$$

This produces for the IIA algebra the same result that we obtained for IIB in the previous section. The closure of the supersymmetry algebra on top-forms at lowest order in the fermions is not enough to guarantee closure at the full level.

\subsection{IIA superspace}

The superspace formulation of IIA supergravity was given in [20], while most of the forms were included in [21, 22]. It was also derived from $D=11$ superspace in [23], from which paper the conventions in this subsection are taken. The Bianchi identities for the RR forms are

$$
d G_{2 n+2}=H_{3} G_{2 n}
$$


while for the NS forms one has

$$
\begin{aligned}
& d H_{3}=0, \\
& d H_{7}=\frac{1}{2} G_{4}^{2}-G_{2} G_{6}, \\
& d H_{9}=-H_{3} H_{7}+\frac{1}{2} G_{4} G_{6}-\frac{3}{2} G_{2} G_{8},
\end{aligned}
$$

The dimension-zero components of the $\mathrm{RR}$ field strengths are proportional to gammamatrices multiplied by $e^{-\phi}$ in the string frame, while for the NS field strengths one has no factor of $e^{-\phi}$ in the case of $H_{3}$, a factor of $e^{-2 \phi}$ for $H_{7}$, while the dimension-zero component of $H_{9}$ vanishes due to the absence of an appropriate symmetric gamma-matrix. The dimension one-half components depend linearly on $\lambda$ with the same dilaton factors $\left(e^{-2 \phi}\right.$ for $\left.H_{9}\right)$.

Now consider the possible eleven-form field strengths. There are two allowable Bianchi identities that can be combined into one:

$$
\begin{aligned}
d H_{11}= & A\left(H_{3} H_{9}+\frac{3}{2} G_{2} G_{10}-\frac{1}{4} G_{6}^{2}\right) \\
& +B\left(-G_{2} G_{10}+G_{4} G_{8}-\frac{1}{2}\right),
\end{aligned}
$$

where $A$ and $B$ are real constants. There are also two possible non-trivial dimension zero components, proportional to $\gamma_{9,2}$ and $\tilde{\gamma}_{9,2}$, where the tilde indicates that a factor of $\gamma_{11}$ is present. The second of these requires that both $A$ and $B$ be zero; we shall come back to this in a moment. For the first case, if we write

$$
H_{9,2}=-i K e^{-2 \phi} \gamma_{9,2}
$$

with $K$ constant, we find that (3.23) is satisfied if $2 A+8 B=K$, so that there are indeed two independent gauge non-trivial eleven-forms. The $(10,1)$ component of $H_{11}$ is proportional to $e^{-2 \phi}(\gamma \cdot \lambda)_{10,1}$ multiplied by a constant depending linearly on $A$ and $B$.

Can there also be a gauge-trivial eleven-form? The answer is yes, but that it is itself trivial, i.e. exact. The dimension-zero component is proportional to $\tilde{\gamma}_{9,2}$, and the dimension one-half component is proportional to $(\tilde{\gamma} \cdot \lambda)_{10,1}$, but the whole form can be written as $d M_{10}$, where the only non-zero component of $M$ is $M_{10,0}=\epsilon_{10,0}$ (i.e. $\epsilon$ regarded as a $(10,0)$-form).

\section{Conclusions}

In this work we re-considered our earlier work [11-13] on top-form potentials in IIA and IIB supergravity. We found in both the IIA and IIB case that the gauge-trivial 10 -form potentials found in our earlier work are excluded by supersymmetry considerations. To be precise, they are allowed by lowest-order supersymmetry but in this work we showed that this is not enough. By considering higher-order fermionic terms we were able to show that gauge-trivial ten-form potentials are forbidden by supersymmetry. The results of this paper are confirmed by an independent (IIA as well as IIB) superspace analysis. Furthermore, 
all gauge non-trivial top-form potentials can be derived by a separate $E_{11 \text {-analysis. This }}$ strongly suggests that we finally obtained full control on the top-form structure of IIA and IIB supergravity.

It remains an open question what the precise brane interpretation is of the different gauge non-trivial top-form potentials. It is known that in the IIB case the D9-brane is part of the quadruplet of 10-form potentials [12]. The situation is less clear for the doublet of eq. (2.26) we found in this work. This doublet does not seem to correspond to a new set of "(p,q) 9-branes" in the usual sense. This can for instance be seen from the fact that it is impossible to write down a kappa-symmetric action for a brane which couples to this 10form potential. The same applies to the IIA case: the two 10-form potentials of eq. (3.20) can not lead to a kappa-symmetric brane effective action. An interpretation of the 10-form potentials as Lagrange multipliers for the constancy of certain gauge parameter functions $g(x)$ seems also out of the question in the absence of any known gauged supergravity in ten dimensions. A similar lack of interpretation exists in the IIA case. This is the least to say intriguing given the fact that most (but not all) other p-forms of IIA and IIB supergravity have a brane interpretation.

It is natural to ask oneself in which sense the results on the top-form structure of maximal ten-dimensional supergravity found in this paper can be extended to other cases with fewer dimensions and/or supersymmetries. In particular, it would be interesting to see whether a general pattern emerges and whether this fits with an extended Kac-Moody algebra structure. These and related questions we leave for future research.

\section{Acknowledgments}

J.H., T.O. and F.R. wish to thank the University of Groningen for its hospitality. The work of J.H. was supported by the Swiss National Science Foundation and the "Innovations- und Kooperationsprojekt C-13" of the Schweizerische Universitätskonferenz SUK/CRUS. The work of T.O. has been supported in part by the Spanish Ministry of Science and Education grant FPA2009-07692, the Comunidad de Madrid grant HEPHACOS P-ESP-00346 and the Spanish Consolider-Ingenio 2010 program CPAN CSD2007-00042. Further, TO wishes to express his gratitude to M.M. Fernández for her permanent support. The work of F.R. was supported by the STFC rolling grant ST/G000/395/1.

Open Access. This article is distributed under the terms of the Creative Commons Attribution Noncommercial License which permits any noncommercial use, distribution, and reproduction in any medium, provided the original author(s) and source are credited.

\section{References}

[1] E. Bergshoeff, M. de Roo, M.B. Green, G. Papadopoulos and P.K. Townsend, Duality of Type II 7-branes and 8-branes, Nucl. Phys. B 470 (1996) 113 [hep-th/9601150] [SPIRES].

[2] L.J. Romans, Massive $N=2 a$ Supergravity in Ten-Dimensions, Phys. Lett. B 169 (1986) 374 [SPIRES]. 
[3] F. Giani and M. Pernici, $N=2$ Supergravity In Ten-Dimensions, Phys. Rev. D 30 (1984) 325 [SPIRES].

[4] I.C.G. Campbell and P.C. West, $N=2 D=10$ Nonchiral Supergravity and Its Spontaneous Compactification, Nucl. Phys. B 243 (1984) 112 [SPIRES].

[5] M. Huq and M.A. Namazie, Kaluza-Klein Supergravity In Ten-Dimensions, Class. Quant. Grav. 2 (1985) 293 [Erratum-ibid. 2 (1985) 597] [SPIRES].

[6] J.H. Schwarz and P.C. West, Symmetries and Transformations of Chiral $N=2 D=10$ Supergravity, Phys. Lett. B 126 (1983) 301 [SPIRES].

[7] J.H. Schwarz, Covariant Field Equations of Chiral $N=2 D=10$ Supergravity, Nucl. Phys. B 226 (1983) 269 [SPIRES].

[8] P.S. Howe and P.C. West, The Complete $N=2, D=10$ Supergravity, Nucl. Phys. B 238 (1984) 181 [SPIRES].

[9] E. Bergshoeff, R. Kallosh, T. Ortín, D. Roest and A. Van Proeyen, New Formulations of D = 10 Supersymmetry and D8-O8 Domain Walls, Class. Quant. Grav. 18 (2001) 3359 [hep-th/0103233] [SPIRES].

[10] E. Bergshoeff, M. de Roo, B. Janssen and T. Ortín, The super D9-brane and its truncations, Nucl. Phys. B 550 (1999) 289 [hep-th/9901055] [SPIRES].

[11] E.A. Bergshoeff, M. de Roo, S.F. Kerstan, T. Ortín and F. Riccioni, IIA ten-forms and the gauge algebras of maximal supergravity theories, JHEP 07 (2006) 018 [hep-th/0602280] [SPIRES].

[12] E.A. Bergshoeff, M. de Roo, S.F. Kerstan and F. Riccioni, IIB Supergravity Revisited, JHEP 08 (2005) 098 [hep-th/0506013] [SPIRES].

[13] E. Bergshoeff, P.S. Howe, S. Kerstan and L. Wulff, Kappa-symmetric $\operatorname{SL}(2, R)$ covariant D-brane actions, JHEP 10 (2007) 050 [arXiv:0708.2722] [SPIRES].

[14] A. Kleinschmidt, I. Schnakenburg and P.C. West, Very-extended Kac-Moody algebras and their interpretation at low levels, Class. Quant. Grav. 21 (2004) 2493 [hep-th/0309198] [SPIRES].

[15] I. Schnakenburg and P.C. West, Kac-Moody symmetries of IIB supergravity, Phys. Lett. B 517 (2001) 421 [hep-th/0107181] [SPIRES].

[16] P.C. West, $E_{11}$, ten forms and supergravity, JHEP 03 (2006) 072 [hep-th/0511153] [SPIRES].

[17] F. Riccioni and P. West, Local E11, JHEP 04 (2009) 051 [arXiv: 0902 .4678] [SPIRES].

[18] G. Dall'Agata, K. Lechner and M. Tonin, $D=10, N=I I B$ supergravity: Lorentz-invariant actions and duality, JHEP 07 (1998) 017 [hep-th/9806140] [SPIRES].

[19] P.C. West, $E_{11}$ and M-theory, Class. Quant. Grav. 18 (2001) 4443 [hep-th/0104081] [SPIRES].

[20] J.L. Carr, S.J. Gates Jr. and R.N. Oerter, $D=10, N=2 a$ Supergravity in Superspace, Phys. Lett. B 189 (1987) 68 [SPIRES].

[21] M. Cederwall, A. von Gussich, B.E.W. Nilsson, P. Sundell and A. Westerberg, The Dirichlet super-p-branes in ten-dimensional type IIA and IIB supergravity, Nucl. Phys. B 490 (1997) 179 [hep-th/9611159] [SPIRES].

[22] E. Bergshoeff, P.M. Cowdall and P.K. Townsend, Massive IIA supergravity from the topologically massive D-2-brane, Phys. Lett. B 410 (1997) 13 [hep-th/9706094] [SPIRES].

[23] P.S. Howe and E. Sezgin, The supermembrane revisited, Class. Quant. Grav. 22 (2005) 2167 [hep-th/0412245] [SPIRES]. 